\title{
Anel intracorneano de Ferrara em ceratocone
}

\author{
Ferrara's intracorneal ring in keratoconus
}

\author{
Hamilton Moreira ${ }^{1}$ \\ Cinara Sakuma de Oliveira ${ }^{2}$ \\ Glaucio de Godoy ${ }^{2}$ \\ Sâmia Ali Wahab ${ }^{2}$
}

\begin{tabular}{|l|}
\hline RESUMO \\
\hline Objetivo: Análise dos resultados dos dez primeiros pacientes com \\
ceratocone submetidos a implante do anel de Ferrara no Hospital de \\
Olhos do Paraná. Métodos: Foi realizado um estudo prospectivo em 10 \\
pacientes com ceratocone. Os critérios de inclusão foram: intolerância a \\
lentes de contato, acuidade visual sem correção inferior ou igual a 20/100, \\
ausência de cicatrizes corneanas significativas e ausência de doenças \\
oculares ou sistêmicas que contra-indicassem a cirurgia. Acuidade \\
visual (LogMAR), refração e topografia pré e pós-operatórias foram as \\
variáveis analisadas. Os pacientes foram seguidos pelo período mínimo \\
de três meses. Resultados: Das complicações cirúrgicas encontradas, \\
destacaram-se dois casos de microperfuração corneana durante a con- \\
fecção das incisões para os túneis inferiores, um de extrusão e quatro de \\
deslocamento pós-operatório do anel. A acuidade visual corrigida me- \\
lhorou de $0,750 \pm 0,374$, para $0,438 \pm 0,342$ (p=0,026). A média da acuidade \\
visual não corrigida no primeiro dia pós-operatório foi de $0,667 \pm 0,447$ \\
(n=9), e a acuidade visual final não corrigida foi de $0,562 \pm 0,272$. De \\
acordo com a refração realizada após o terceiro mês pós-operatório, cinco \\
pacientes evoluíram com acuidade visual com correção melhor do que ou \\
igual a 0,5 unidades LogMAR. Um paciente não apresentou aplanamento \\
significativo do cone segundo a topografia corneana. Conclusão: O \\
presente estudo demonstrou melhora nos valores pós-operatórios de \\
acuidade visual, refração e topografia na maioria dos pacientes avalia- \\
dos, em relação aos valores pré-operatórios.
\end{tabular}

Descritores: Ceratocone/cirurgia; Estroma corneal/cirurgia; Próteses e implantes; Acuidade visual; Topografia da córnea; Estudos prospectivos

\section{INTRODUÇÃO}

O ceratocone é uma ectasia corneana, geralmente bilateral e assimétrica, com uma incidência de um caso entre 2000 indivíduos na população geral. As lentes de contato rígidas gás-permeáveis são a melhor opção terapêutica, porém geralmente torna-se necessária a realização da ceratoplastia penetrante. A taxa de transparência do botão após os transplantes é maior do que $90 \%$, no entanto, várias complicações são relacionadas a esta cirurgia como: falência primária ou secundária, rejeição, ceratites infecciosas, desepitelização persistente e principalmente alto astigmatismo ${ }^{(1)}$.

Anéis intracorneanos para correção do ceratocone podem ser uma alternativa para estes pacientes que não toleram o uso de lentes de contato e que não desejam enfrentar os riscos de um transplante de córnea.

A utilização de implantes intracorneanos para a correção de ametropias é uma prática antiga, descrita por alguns autores no início da década de $60^{(2-3)}$. Barraquer foi o primeiro a realizar implantes intracorneanos em animais, 
utilizando diversos materiais, com diâmetros e curvaturas variáveis, observando como principais complicações as freqüientes extrusões ou alterações na região anterior do implante. Inicialmente desenvolvidos para a correção de miopias leves a moderadas, os anéis intraestromais apresentaram como vantagens a maior previsibilidade dos resultados e o maior controle da reação cicatricial devido a biocompatibilidade dos implantes utilizados ${ }^{(2)}$.

Os implantes intracorneanos têm sido utilizados seguindo a "Lei da Espessura" de Barraquer: "Toda vez que um tecido for adicionado à periferia ou removido do centro da córnea, um correspondente aplanamento é obtido e vice-versa"(2). Em 1986, foi desenvolvido o anel corneano intraestromal (ACI) chamado de Anel de Ferrara, que foi utilizado pela primeira vez em 1991, em um paciente amblíope, e tem sido indicado pelo autor para a correção de miopia até 15 dioptrias, ceratocone e astigmatismo irregular após transplante de córnea ${ }^{(4)}$.

O presente estudo objetiva analisar os resultados de acuidade de visual, refração e topografia dos 10 primeiros pacientes com ceratocone submetidos a implante do anel de Ferrara no Hospital de Olhos do Paraná.

\section{MÉTODOS}

Dez pacientes com diagnóstico de ceratocone foram selecionados de acordo com os critérios de inclusão e exclusão estabelecidos e operados em estudo clínico prospectivo. Foram incluídos neste estudo pacientes portadores de ceratocone com acuidade visual sem correção inferior a 20/100 e intolerância a lentes de contato. Os critérios de exclusão foram: doenças sistêmicas como diabetes, doenças do colágeno e metabólicas, presença de qualquer doença ocular e leucoma após hidropsia que comprometesse a transparência corneana. Todos os pacientes assinaram termo de consentimento específico, estando cientes da característica experimental da técnica cirúrgica proposta.

Foram realizados exames pré-operatórios incluindo: exame oftalmológico, topografia corneana (EyeSys) e paquimetria (Sonomed), sendo esta realizada no local onde a córnea apresentava o eixo mais curvo, segundo a topografia. Foi realizada a equivalência das medidas da acuidade visual entre as escalas de Snellen e LogMAR, para a comparação entre os resultados pré e pós-operatório. O olho não dominante do paciente foi selecionado e operado sob anestesia tópica, exceto em uma paciente com Síndrome de Down que foi submetida à anestesia geral.

$\mathrm{O}$ anel utilizado em todos os pacientes consistia em dois segmentos compostos de PMMA, com espessura variando de 100 a 350 micra dependendo do grau do ceratocone (Quadro 1) e com dois orifícios nas extremidades. Cada segmento possuía comprimento de 150 graus de arco e diâmetro de ápice de cinco milímetros, apresentando uma secção triangular, cuja base era constante para todas as espessuras.

Foi marcado o centro óptico corneano com o paciente

\begin{tabular}{|c|c|}
\hline \multicolumn{2}{|c|}{ Quadro 1. Nomograma para escolha da espessura do anel } \\
\hline Espessura do anel (em micra) & Grau evolutivo do ceratocone \\
\hline 200 & I - até $45 \mathrm{D}$ \\
\hline 250 & II - de 45 a 52 D \\
\hline 300 & III - de 52 a 60D \\
\hline 350 & IV - acima de 60D \\
\hline
\end{tabular}

olhando para a luz e entre 2,5 e 3,5 mm deste ponto, foram realizadas duas incisões radiais em direções opostas no mesmo eixo, nos locais onde a topografia corneana era mais curva. As incisões foram realizadas com bisturi de diamante sob orientação da paquimetria, atingindo $80 \%$ da espessura corneana. Pelas incisões foi introduzido um tunelizador circular duplo que foi rodado 180 graus no sentido horário para confecção simultânea de dois túneis corneanos estromais. Em seguida, um dilatador circular foi utilizado para aumentar estes túneis e os dois segmentos de anel foram introduzidos. Caso a incisão ficasse um pouco aberta, era confeccionado um ponto corneano com nylon $10.0 \mathrm{com}$ agulha de transplante. Os instrumentos cirúrgicos foram desenvolvidos especialmente para esta técnica cirúrgica (Ferrara Ophthalmics).

No pós-operatório foi prescrito colírio de associação de dexametasona $0,1 \%$ e tobramicina $0,3 \%$ seis vezes ao dia na primeira semana e quatro vezes ao dia por mais seis semanas, e anti-inflamatório não hormonal na forma de colírio (cetorolac $0,5 \%$ ) nas primeiras 24 horas. As suturas de córnea foram removidas em consultório nos primeiros 15 dias. Os pacientes foram avaliados no $1^{\circ}, 3^{\circ}, 7^{\circ}$ e $15^{\circ}$ dias e no $1^{\circ} \stackrel{\circ}{2} 2^{\circ}$ e $3^{\circ}$ meses. Os seguintes exames foram realizados no pós-operatório: acuidade visual de Snellen, cujos resultados foram posteriormente transformados em valores equivalentes, segundo a escala de LogMAR, refração sem cicloplegia, topografia corneana, biomicroscopia e tonometria de aplanação.

\section{RESULTADOS}

Dos 10 pacientes operados, cinco eram do sexo feminino e cinco do masculino, com idades variando de 15 a 56 anos (média de 27,4 anos), todos pertencentes à raça branca. A acuidade visual pré-operatória com e sem a melhor correção com óculos está mostrada na tabela 1.

\section{Complicações:}

Todas as cirurgias foram realizadas com anestesia tópica exceto uma, na qual o paciente foi submetido à anestesia geral por apresentar Síndrome de Down. Dois pacientes apresentaram microperfuração corneana no momento de uma das incisões, sendo necessário o preenchimento da câmara anterior com viscoelástico (ácido hialurônico) devido à hipotonia ocasionada, prosseguindo-se com a implantação do anel.

Quatro pacientes relataram leve desconforto no primeiro pós-operatório e cinco não apresentaram nenhuma queixa. $\mathrm{O}$ 


\begin{tabular}{|lccc|}
\hline \multicolumn{4}{|c|}{$\begin{array}{c}\text { Tabela 1. Acuidade visual (LogMAR) com e sem correção e } \\
\text { refração pré-operatórias dos dez pacientes estudados }\end{array}$} \\
Paciente & AV & Refração & AV \\
& sem correção & & com correção \\
1 & OE $-1,3$ & $+2,00-5,5095^{\circ}$ & 0,3 \\
$2^{*}$ & OE $-1,9$ & xxxx & Xxxx \\
3 & OE $-1,9$ & $-9,00$ & 1,0 \\
4 & OD $-1,0$ & $-3,50-2,50170^{\circ}$ & 0,5 \\
5 & OD $-0,7$ & $\mathrm{PL}-2,5075^{\circ}$ & 0,3 \\
6 & OD $-0,7$ & $-7,00-2,5090^{\circ}$ & 0,3 \\
7 & OD $-1,9$ & $-4,50-4,0090^{\circ}$ & 1,0 \\
8 & OD $-1,9$ & $-4,00-1,0040^{\circ}$ & 1,0 \\
9 & OD $-1,9$ & $-7,50-6,0030^{\circ}$ & 1,3 \\
10 & OD $-0,7$ & $-3,00-5,5045^{\circ}$ & 0,6 \\
${ }^{*}$ paciente com S. de Down. & & \\
\hline \multicolumn{5}{r}{} \\
\hline
\end{tabular}

paciente com Síndrome de Down não permitiu uma avaliação pós-operatória adequada. Um dos pacientes apresentou extrusão da prótese com sete dias de pós-operatório. Devido ao grande edema corneano no local, optou-se por reimplantar o anel mais tarde, através de uma nova incisão. Outro paciente apresentou deslocamento do anel com início de extrusão no 15 dia e como não apresentava edema de córnea ou qualquer sinal de reação inflamatória, decidiu-se por reposicioná-lo, retirando-o e aumentando o túnel. Três pacientes apresenta- ram deslocamento do anel, aproximando-se da incisão, mas sem sinal de extrusão, na qual a conduta foi a observação. Um dos pacientes com microperfuração intra-operatória, apresentou crescimento de células epiteliais em todo o túnel por volta do $30^{\circ}$ dia de pós-operatório. Não ocorreram casos de ceratite infecciosa ou neovascularização corneana.

\section{Acuidade visual:}

Os resultados da acuidade visual sem correção obtidos no pós-operatório estão dispostos na tabela 2. Houve melhora da acuidade visual sem correção em $70 \%$ dos pacientes no primeiro dia de pós-operatório, quando comparada com os resultados do pré-operatório. A acuidade visual não corrigida melhorou de $1,322 \pm 0,585$ para $0,562 \pm 0,272$, sendo $p=0,003$.

A refração foi realizada após o $3^{\circ}$ mês devido à instabilidade da acuidade visual. Cinqüenta por cento dos pacientes evoluíram com acuidade visual com correção melhor ou igual a 0,5 unidades LogMAR, como mostra a tabela 3. A acuidade visual corrigida melhorou de $0,750 \pm 0,374$ para $0,438 \pm 0,342$, sendo $\mathrm{p}=0,026$.

Nove pacientes apresentaram aplanamento acentuado do cone pela topografia, como mostra a tabela 4 .

Em razão de a amostra analisada ser muito pequena, podese notar que existe uma tendência para que, quanto maior for a espessura do anel implantado, maior será o aplanamento obtido, conforme demonstrado no Gráfico 1. Ressalta-se portanto,

Tabela 2. Acuidade visual (sem correção) do primeiro dia ao terceiro mês de pós-operatorio entre os dez pacientes estudados

\begin{tabular}{|c|c|c|c|c|c|c|c|c|}
\hline Paciente & AV pré & 1 Dia & 3 Dia & $7^{\circ} \mathrm{Dia}$ & 15 Dia & $1^{\circ}$ Mês & 2으s & 3ำ Mês \\
\hline 1 & 1,3 & 0,2 & 0,5 & 0,6 & XXX & $X X X$ & $\mathrm{XXX}$ & 0,5 \\
\hline 2 & 1,9 & $x x x$ & $x x x$ & $x x x$ & $x x x$ & $x x x$ & $x x x$ & $x x x$ \\
\hline 3 & 1,9 & 0,5 & 0,6 & 0,6 & 0,6 & 0,6 & 0,7 & 1,0 \\
\hline 4 & 1,0 & 1,0 & 1,0 & 1,0 & 1,0 & 1,0 & 0,7 & 0,7 \\
\hline 5 & 0,6 & 0,2 & 0,2 & 0,2 & 0,2 & 0,2 & 0,2 & 0,2 \\
\hline 6 & 0,7 & 1,3 & 1,3 & EXTR. * & & & & \\
\hline 7 & 1,9 & 0,6 & 0,6 & 0,6 & $0,6^{\star *}$ & 0,2 & 0,2 & 0,2 \\
\hline 8 & 1,9 & 1,3 & 1,0 & 1,3 & 1,3 & 1,9 & 1,0 & 0,7 \\
\hline 9 & 1,9 & 0,7 & 0,7 & 1,3 & 1,0 & 1,0 & 0,7 & 0,7 \\
\hline 10 & 0,7 & 0,2 & 0,2 & 0,2 & 0,5 & 0,6 & 0,5 & 0,5 \\
\hline
\end{tabular}

\begin{tabular}{|c|c|c|c|c|}
\hline Paciente & Refração Pré & AV c/c Pré & Refração Pós & AV c/c Pós \\
\hline 1 & $+2,00-5,0095^{\circ}$ & 0,3 & $\mathrm{pl}-1,0015^{\circ}$ & 0,5 \\
\hline 2 & $\mathrm{XXX}$ & $x x x$ & $\mathrm{XXX}$ & $x x x$ \\
\hline 3 & $-9,00$ & 1,0 & $-2,25-3,50165^{\circ}$ & 1,0 \\
\hline 4 & $-3,50-2,50170^{\circ}$ & 0,5 & $-5,00-4,5030^{\circ}$ & 0,2 \\
\hline 5 & $\mathrm{pl}-2,5075^{\circ}$ & 0,3 & $\mathrm{pl}-2,0045^{\circ}$ & 0,0 \\
\hline 6 & $-7,00-2,5090^{\circ}$ & 0,3 & $x x x$ & $x x x$ \\
\hline 7 & $-4,50-4,0090^{\circ}$ & 1,0 & $+1,00-1,00135^{\circ}$ & 0,2 \\
\hline 8 & $-4,00-1,0040^{\circ}$ & 1,0 & $-2,00-4,0030^{\circ}$ & 0,7 \\
\hline 9 & $-7,50-6,0030^{\circ}$ & 1,3 & $-3,00-2,00180^{\circ}$ & 0,7 \\
\hline 10 & $-3,00-5,5045^{\circ}$ & 0,6 & $-2,00-4,0090^{\circ}$ & 0,2 \\
\hline
\end{tabular}




\begin{tabular}{|c|c|c|c|c|c|c|}
\hline Paciente & Ápice Cone Pré & Espessura do anel & $10 \mathrm{Dia}$ & 1ำ Mês & 3ํMês & Aplanamento Obtido \\
\hline 5 & OD 49,50 & 150 & - & 41,50 & 41,50 & 8,00 \\
\hline 1 & OE 63,13 & 250 & 55,88 & - & 55,73 & 7,40 \\
\hline 2 & OE 59,28 & 250 & 51,15 & - & 43,44 & 15,84 \\
\hline 4 & OD 51,50 & 250 & 56,19 & 56,50 & 53,33 & $-2,86$ \\
\hline 9 & OD 50,80 & 250 & 46,50 & 45,78 & 45,80 & 5,00 \\
\hline 3 & OE 61,23 & 300 & 47,00 & 48,86 & 46,90 & 14,33 \\
\hline 6 & OD 64,00 & 300 & 56,00 & - & - & - \\
\hline 7 & OD 66,00 & 300 & 53,00 & 53,00 & 52,51 & 13,49 \\
\hline 10 & OD 57,00 & 300 & 46,45 & 47,00 & 46,44 & 10,56 \\
\hline 8 & OD 74,00 & 350 & 65,21 & 67,82 & 65,30 & 8,70 \\
\hline
\end{tabular}

\begin{tabular}{|c|c|c|c|c|c|c|}
\hline Espessura do anel & № de Pacientes & Média & Desvio Padrão & Mínimo & Máximo & Mediana \\
\hline 150 & 01 & 8,0 & - & - & - & - \\
\hline 250 & 04 & 6,3 & (1) 7,7 & $-2,9$ & 15,8 & 6,2 \\
\hline 300 & 03 & 12,8 & 2,0 & 10,6 & 14,3 & - \\
\hline 350 & 01 & 8,7 & - & - & - & - \\
\hline TOTAL & 09 & 8,9 & (1) 5,7 & $-2,9$ & 15,8 & 8,7 \\
\hline
\end{tabular}

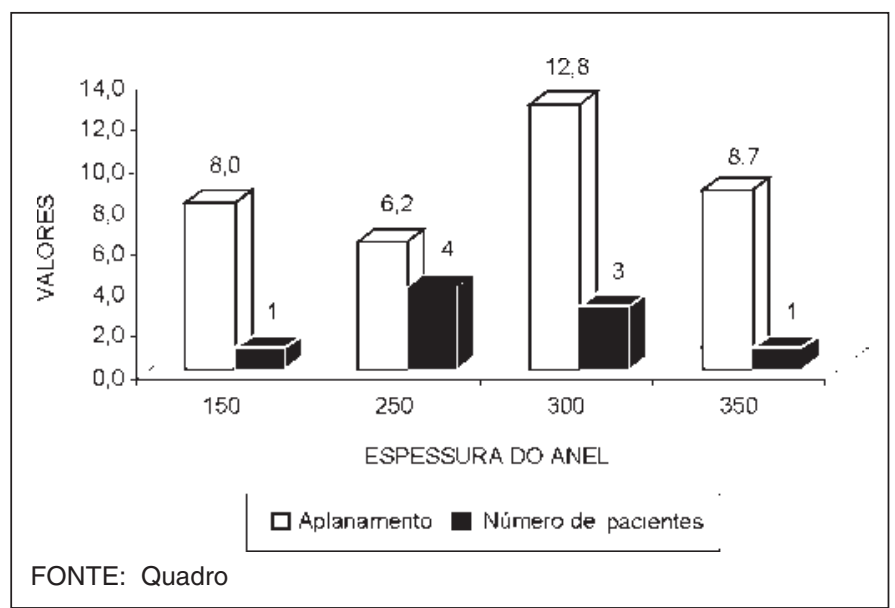

Gráfico - Relação entre os valores do aplanamento do cone e a espessura do anel intracorneano utilizado em nove procedimentos cirúrgicos

que nada pode ser concluído e nenhum teste estatístico pode ser aplicado.

Não houve alteração significativa na pressão ocular em nenhum paciente após a cirurgia.

\section{DISCUSSÃO}

Estudos referentes à utilização de anéis intracorneanos para a correção de ceratocone têm sido muito freqüentes nos últimos anos. A cada dia esta técnica torna-se uma solução mais atraente aos pacientes cuja última alternativa era o transplante penetrante de córnea. Dentre as principais vantagens da cirurgia destaca-se a preservação da asfericidade positiva da córnea e conservação da sua porção central e o seu caráter reversível. Além disso, nada impede que se realize o transplante penetrante caso a cirurgia do anel não alcance o resultado esperado $^{(4-5)}$.

Das 10 cirurgias realizadas, duas apresentaram como intercorrência intra-operatória microperfurações corneanas que ocorreram durante a confecção das incisões inferiores. As incisões muito superficiais levam à formação de um túnel muito superficial, com maior facilidade de extrusão do anel. Pelo fato de o cone levar ao afinamento corneano exatamente coincidente ao local onde se realiza uma das incisões para a confecção dos túneis, esta técnica cirúrgica apresenta alto grau de dificuldade. Estas intercorrências, porém, não interferiram no bom andamento cirúrgico, permitindo a realização do túnel e implantação dos anéis após o preenchimento da câmara anterior com viscoelástico.

Das complicações pós-operatórias, houve um caso de extrusão do anel, um de crescimento epitelial no túnel e quatro de deslocamento do anel, sendo necessário reposicionamento em um deles. As complicações mais freqüentes descritas após o implante de anel intraestromal, tem sido relacionadas a erros de técnica ${ }^{(4,6)}$. Portanto, das 10 cirurgias duas apresentaram intercorrências per-operatórias e cinco evoluíram com complicações pós-operatórias. A curva de aprendizado desta técnica talvez tenha sido responsável por algumas destas complica- 
ções. Talvez com a pressurização do globo durante a confecção do túnel, seja possível evitar algumas destas complicações.

O sintoma mais freqüente no pós-operatório imediato foi desconforto leve, que foi bem controlado com a medicação. No pós-operatório tardio, a queixa mais freqüente foi fotofobia e oscilação da acuidade visual. Estudos relatam que sintomas como ofuscamento e fotofobia tendem a ser limitados ${ }^{(4)}$.

A melhora da acuidade visual não corrigida, nos primeiros dias de pós-operatório tem sido a regra e está relacionada à diminuição dos erros refrativos e à preservação da asfericidade positiva da córnea ${ }^{(4,6)}$. Os dados encontrados na literatura referentes à variação da acuidade visual foram confirmados neste estudo. Em geral, a sua estabilização ocorre após o $3^{3}$ mês ${ }^{(4,6-7)}$.

$\mathrm{O}$ aplanamento corneano induzido pela cirurgia e evidenciado em nove pacientes através da topografia de córnea, tem sido relatado por outros autores ${ }^{(8-9)}$. Não houve correlação entre a espessura do anel e o aplanamento do ápice do cone. Talvez a falta de precisão no posicionamento e na profundidade dos anéis explique este fato. A acuidade visual melhorou em todos os casos nos quais obteve-se aplanamento do ápice corneano, sendo que dois pacientes atingiram 0,2 unidades LogMAR sem correção e cinco alcançaram 0,5 ou melhor com correção (óculos).

\section{CONCLUSÃO}

Apesar do alto índice de complicações, este estudo demonstrou melhora da acuidade visual com e sem correção e aplanamento corneano significativo após a implantação dos anéis. Mais investigação e um acompanhamento mais longo destes pacientes se faz necessário.

\section{ABSTRACT}

Purpose: To evaluate the results of the first 10 keratoconus patients submitted to Ferrara's ring surgery. Methods: In a prospective study, 10 keratoconus patients were included with best uncorrected visual acuity equal to or worse than 20/100, contact lens intolerance and absence of significant corneal scar. Patients were healthy otherwise, with no systemic medications or diseases. LogMAR visual acuity, refraction, topography and surgical complications were recorded. Minimum follow up was three months. Results: Two microperfurations occurred during the incision for the inferior tunnel. After surgery we had one case of single acrylic extrusion and four cases of displacement of one or both segments. Overall spectacle corrected visual acuity improved from $0.750 \pm 0.374$, to $0.438 \pm 0.342(\mathrm{p}=0.026)$. The mean noncorrected visual acuity on the first day after surgery was $0.667 \pm 0.447(n=9)$, and final noncorrected visual acuity of $0.562 \pm 0.272$ was recorded. Five patients achieved spectacle corrected visual acuity of 0.5 LogMAR units was recorded or better, after three months of surgery. One patient did not show any significant flattening of the cornea at topography. Conclusion: This study showed improvement of visual acuity, refraction and topography in most patients in relation to preoperative results.

Keywords: Keratoconus/surgery; Corneal stroma/surgery; Prostheses and implants; Visual acuity; Corneal topography; Prospective studies

\section{REFERÊNCIAS}

1. Schor P. Ceratocone. Arq Bras Oftalmol 1998;61:235-7.

2. Barraquer JI. Modification of refraction by means of intracorneal inclusion. Int Ophthalmol Clin 1966;6:53-78.

3. Belau PG, Dyer JA, Ogle KN, Henderson JW. Correction of ametropia with intracorneal lenses: an experimental study. Arch Ophthalmol 1964;72:541.

4. Nosé W, Ferrara P. Anillo intracorneal. In: Albertazzi R, Centurion V. La moderna cirurgia refrativa. Buenos Aires: Gustavo Multedo; 1999. p.167-81.

5. Holmes-Higgin DK, Baker PC, Burris TE, Sivestrini TA. Characterization of the aspheric corneal surface with intrastromal corneal ring segments. J Refract Surg 1999; $15: 520-8$.

6. Nosé W, Neves RA, Burris TE, Schanzlin DJ, Belfort Jr. R. Intrastromal corneal ring: 12-month sighted myopic eyes. J Refract Surg 1996;12:20-8.

7. Barach D, Mortemousque B, Bertel F, Diemer C, Dorot N, Verin P. Fluctuations in uncorrected visual acuity after refractive surgery using intrastromal corneal rings. J Fr Ophthalmol 1999;22:335-8.

8. Burris TE, Ayer CT, Evensen DA, Davenport JM. Effects of intrastromal corneal ring size and thickness on corneal flattening in human eyes. Refract Corneal Surg 1994;7:45-50.

9. Burris TE, Baker PC, Ayer CT, Loomas BE, Mathis L, Silvestrini T. Flattening of central corneal curvature with intrastromal corneal ring of increasing thickness: an eye bank eye study. J Cataract Refract Surg 1993;19 (suppl):182-7.

\title{
ABO ELETRÔNICO
}

\author{
Novo site
}

\section{Acesso: http://www.abonet.com.br}

